\title{
Enfermedad de la mucosa oral: Penfigoide de las membranas mucosas
}

\section{Oral mucosal disease: Mucouse membrane pemphigoid}

\author{
Discepoli N*, Bascones Martínez A**
}

\section{RESUMEN}

Los trastornos vesículobullosos subepiteliales representan desordenes autoinmunitarios que cogen origen de reacciones dirigidas hacia componentes de los hemidesmosomas o bien de las Zonas de la Membrana Basal (ZMB) de los epitelios escamosos estratificados. A estos trastornos ha sido conferido el término de enfermedades ampollosa subepiteliales inmunomediadas (EASIM) y el penfigoide de las membranas mucosas (PMM) es el más frecuente.

Todas las enfermedades subepiteliales vesiculobullosas se presentan como lesiones ampollosas y descamativas, y el diagnostico debe de ser confirmado por una biopsia junta a tinción inmunológica.

No hay un único tratamiento capaz de controlar todas las enfermedades subepiteliales vesiculoampollosas; las diferencias inmunológicas entre las EASIM proporciona diferencias en las respuestas al tratamiento. Hoy en día el tratamiento inmunorregulador es usado para controlar la lesión oral de PMM.

Palabras clave: Penfigoide, mucosas orales, autoinmunidad, corticosteroides, inmunosupresores, oral.

\section{SUMMARY}

Subepithelial vesiculobullous conditions are chronic autoimmune disorders that arise from reactions directed against components of the hemidesmosomes or basement membrane zones (BMZ) of stratified squamous epithelium to which the term immune-mediated subepithelial blistering diseases (IMSEBD) has been given. Mucous membrane pemphigoid (MMP) is the most common, but variants do exist. All subepithelial vesiculobullous disorders present as blisters and erosions, and diagnosis must be confirmed by biopsy examination with immunostaining, sometimes supplemented by other investigations. No single treatment reliably controls all subepithelial vesiculobullous disorders; the immunological differences within IMSEBD may account for differences in responses to treatment. Currently, as well as improving oral hygiene, immunomodulatory treatment is used to control the oral lesions of MMP, but it is not known if its specific subsets reliably respond to different agents.

Key words: Pemphigoid, skin, autoimmune, corticosteroids, immunosuppressants, oral.

Fecha de recepción: 16 de octubre de 2009.

Aceptado para publicación: 20 de octubre de 2009.

* $\quad$ Máster de Periodoncia e Implantes. Universidad Complutense. Madrid.

** Catedrático de Medicina Bucal y Periodoncia. Universidad Complutense. Madrid. Director del Máster de Periodoncia.

Discepoli N, Bascones Martínez A. Enfermedad de la mucosa oral: Penfigoide de las membranas mucosas. Av. Odontoestomatol 2009; 25 (2): 59-66. 


\section{INTRODUCCIÓN}

Las primeras enfermedades bullosas identificadas fueron el pénfigo y la dermatitis herpetiforme; muchos trastornos vesiculobullosos fueron reconducidos bajo estas definiciones (1-2). El termino penfigoide fue usado por primera vez cuando se identificaron a lo largo de la ZMB de epitelios escamosos estratificados depósitos de inmunoglobulinas ( $\operatorname{lgG}$ y C3) (2). La enfermedad sigue un decurso menos severo respecto al pénfigo. De todas formas muy pronto fue evidente que el penfigoide representaba una familia de enfermedades ampollosas subepiteliales inmunomediadas que incluya: penfigoide bulloso, penfigoide gestationis, penfigoide cicatricial, dermatitis herpetiforme, y la enfermedad linear de IgA. Esta heterogeneidad condujo a la formulación del término Enfermedades Ampollosas Subepiteliales Inmunomediadas (EASIM), y se reconoció que en el penfigoide auto anticuerpos IgG pueden dirigirse hacia varios antígenos de la ZMB (5-7). En las muestras tomada con biopsia de lesiones de penfigoide se puede evidenciar la mayoría de los autoanticuerpos presentan depósitos que se localizan a nivel del piso de la ZMB (salt-split deposits). El término genérico penfigoide incluye el penfigoide cicatricial (actualmente renombrado Penfigoide de las Membranas Mucosas [PMM]), penfigoide bulloso, penfigoide gestationis, anti p-200, anti p-105 y penfigoide anti $\mathrm{p}-450$, penfigoide lichen planus, dermatitis herpetiforme, enfermedad linear de IgA, epidermólisis bullosa adquirida, y pénfigo paraneoplásicos (8-9). De todas formas todavía no queda claro cuánto el PMM sea heterogéneo por sí mismo (34).

\section{PENFIGOIDE DE LAS MEMBRANAS MUCOSAS}

El fenotipo que caracteriza el PMM consiste en vesículas, erosiones, o ambas suele afectar las mucosas, frecuentemente la mucosa oral. Se presenta más frecuentemente en mujeres, con una edad de comienzo alrededor de 51-62 años (8).

El PMM no es una entidad única desde el punto de vista clínico, ni tampoco desde el punto de vista inmunológico; recientemente avances a nivel de biología molecular han revelado su heterogeneidad. Hasta la fecha 10 diferentes componentes de la ZMB han sido identificados como posibles autoantígenos in distintas EASIM; se suele asociar frecuentemente al PMM el antígeno 2 del penfigoide bulloso (BPAg2) y con menos frecuencia al antígeno 1 del penfigoide bulloso (BPAg 1), a la lámina 5 (epilegrina), al colágeno de tipo VII y por último a la subunidad $\beta 4$ de la integrina $\alpha 6 \beta 4$ (10-11).

Estas variaciones en antígenos y anticuerpos podría reflejarse en variaciones en el mecanismo patogenético, en la presentación clínica y en la terapia.

\section{ETIOLOGÍA Y PATOGENIA}

El factor de iniciación para la respuesta autoinmunitaria es desconocido, aunque una droga como la furosamida puede ocasionalmente representar el primus movens de dicha reacción (12). También se ha postulado una predisposición genética, con una asociación con HLA DBQ1*0301, que puede tener un papel en el reconocimiento de los antígenos en la ZMB por parte de los linfocitos T (11).

En el PMM los inmunodepósitos que reflejan la reacción de los autoanticuerpos pueden encontrarse entre la zona de la membrana basal y el epitelio, y están compuestos por IgG $(97 \%)$ con C3 (78\%) en la mayoría de los casos; también pueden hallarse IgA y $\lg M(27 \%$ y $12 \%)$, hecho que confirmaría la heterogeneidad de los antígenos responsables de la reacción autoinmunitaria en el PMM (14-15).

La patogenia del PMM incluye un secuestro de leucocitos (primariamente neutrófilos) inducida por autoanticuerpos y mediada por los factores del complemento. El resultado es una liberación de citoquinas y enzimas leucocitarios, con el desprendimiento de las células basales de la zona de la membrana basal (16). Se puede asistir también a lisis células mediada por factores del complemento.

\section{VARIANTES DEL PMM CON LESIONES ORALES}

Se han clasificado como mínimo seis variantes de PMM con diferente especificidad de autoanticuerpos y diferentes patrones de inmunopatogenia. Las principales variantes orales son las siguientes. 


\section{Penfigoide Oral: PMMO (sólo lesiones orales)}

El antígeno diana todavía no está claro, aunque anticuerpos contra una proteína de membrana de 168$\mathrm{kDa}$ han sido hallados en algunos pacientes y según algunos autores, de alguna forma, estaría involucrada como antígeno una variante de la $\alpha 6$ integrina (17). El penfigoide oral presenta una sensibilidad muy baja al análisis con inmunofluorescencia indirecta o con técnicas serológicas en respecto a los antígenos del penfigoide bulloso o a los otros antígenos más comunes en los casos de PMM (18).

\section{Penfigoide con características clínicas más extensas (lesiones orales y extraorales)}

Esta entidad clínica está asociada con una reactividad de autoanticuerpos hacia múltiples antígenos de la zona de la membrana basal. El penfigoide cicatricial anti-epilegrina es una importante entidad, que es indistinguible desde el punto de vista clínico, de los otros penfigoides cicatriciales (18). Suele interesar la boca, los ojos y la piel y está caracterizada en el examen con microscopia a inmunofluorescencia por la unión de IgG a la laminina 5, como antígeno diana. Esta unión se produce de manera masiva hacia el lado dérmico de la membrana basal (salt-split human skin).

\section{CARACTERÍSTICAS CLÍNICAS}

El fenotipo clínico conocido como PMM pues no es una entidad única; incluye pacientes solamente con lesiones orales, con lesiones orales y de otras mucosas y de la piel y otras variantes pueden tener complicaciones sistémicas. La presencia de lesiones orales suele presentar secuelae muy graves; cuando la lesión ocular está limitada a la conjuntiva, se usa el termino penfigoide cicatricial ocular (19).

\section{Lesiones orales}

La mucosa oral es muchas veces el lugar de inicio de la lesión en distintas variantes del PMM. Los pacientes presentan disfagia, dolor y descamación de la mucosa. Vesículas y bullas pueden desarrollarse en cualquier zona de la mucosa oral (Figs. 1 a 4), y

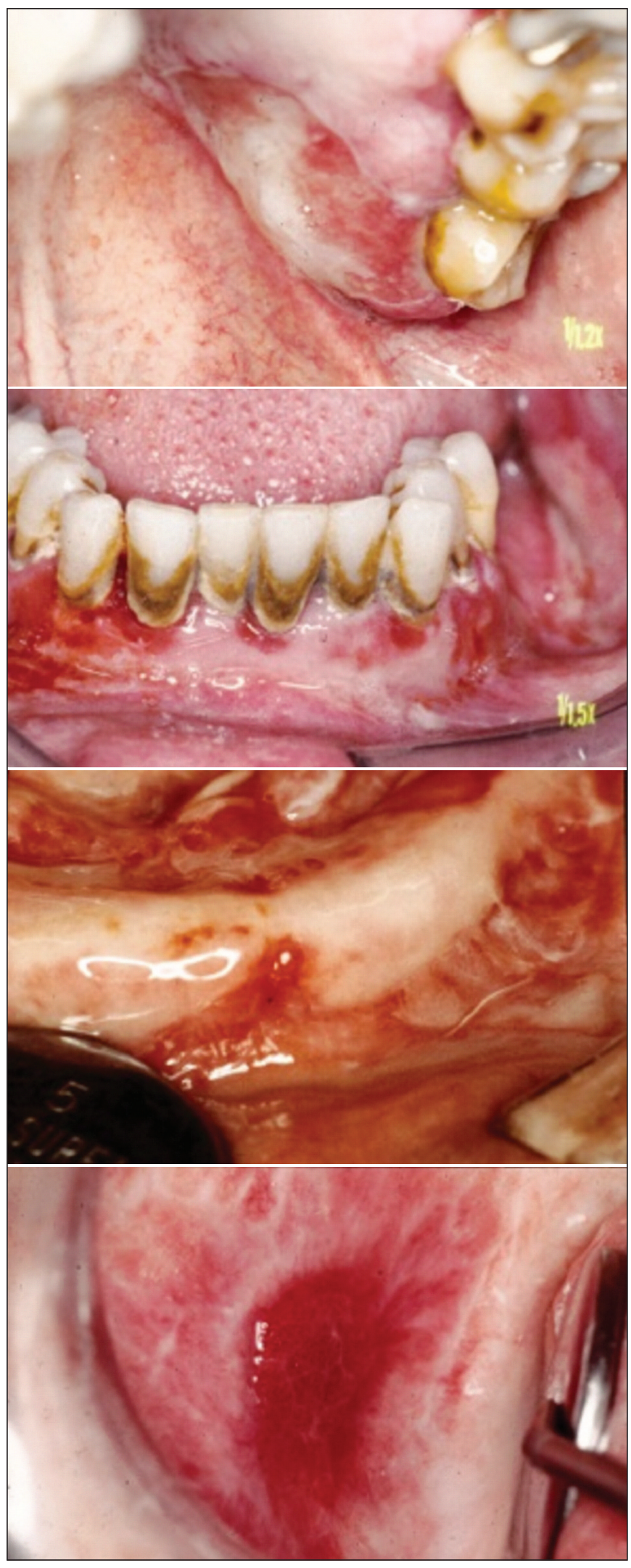

Figs. 1 a 4 . 
el signo de Nikolsky puede ser positivo (como para el pénfigo) (20) .Cuando ocurre la ruptura de estas lesiones iníciales lo que queda es una erosión de forma irregular con la presencia de un alón inflamatorio y de exudado amarillento. Los pacientes con PMM presentan muy a menudo lesiones orales (20).

El PMM es la principal causa de la gingivitis descamativa, y esta última representa la principal característica oral del mismo pénfigo y una de las lesiones de aparición más temprana. El grado de descamación puede variar considerablemente; desde pequeñas áreas, hasta un eritema muy extenso, con superficie reluciente. Esta condición se une a un estado de dolor crónico. Estos pacientes suelen presentar una acumulación de placa superior a los controles y consecuente inflamación gingival; se pueden hallar en estos pacientes también más recesiones y mas lesiones furcales.

\section{Otras lesiones}

Las lesiones pueden dirigirse hacia otros epitelios escamosos estratificados, como aquellos de los ojos, de la laringe, del esófago, de la nariz, de la vulva, del pene o del ano. Acerca del $40 \%$ de los pacientes con PMM pueden desarrollar una complicación a nivel ocular. La patología ocular empieza con una conjuntivitis crónica con síntomas como quemazón, irritación, fotofobia. Los síntomas interesan inicialmente un solo ojo y si se dejan sin tratar entro un tiempo variable de dos años, pueden interesar los dos. Las cicatrices pueden conducir a la fusión de las pálpebras superiores e inferiores (ankioblefaron).

\section{Lesiones de la piel}

La presencia de lesiones epidérmicas no es un hecho muy común (hasta un cuarto de los pacientes) y está confinada a la piel de la cara, del cuello, de las axilas y de las extremidades.

\section{Asociación con enfermedades sistémicas}

El PMM se puede asociar ocasionalmente con otros trastornos autoinmunes como el pénfigo (22). Algunos casos de penfigoide cicatricial han sido asocia- dos con desordenes linfoproliferativos de las células B. Pacientes con penfigoide cicatricial deantiepilegrina poseen una incidencia mayor de cánceres sólidos, respecto a la población sana (23).

\section{DIAGNÓSTICO}

La presencia de rupturas de bullas que dejan erosiones de forma irregulares y el signo de Nikolsky positivo, no se pueden considerar específicos para ninguna forma de penfigoide (25). El principal diagnóstico diferencial incluye lichen planus, pénfigo, eritema multiforme y el lupus eritematoso sistémico (26).

El diagnóstico tiene que confirmarse con el uso de técnicas histológicas y de inmunofluorescencia directa sobre muestra de tejido obtenidas con biopsia. Es muy importante biopsiar una vesículas o del tejido cerca la lesión; en general no está indicado biopsiar un erosión, porque representa la perdida de tejido que sería interesante analizar.

Histológicamente el PMM está caracterizado por una separación del epitelio a nivel de la ZMB, que genera una división subbasilar, con la presencia de un infiltrado inflamatorio en la lámina propia, con presencia de eosinófilos, linfocitos y neutrófilos (Figs. 5 y 6). La identificación con inmunofluorescencia directa de depósitos de IgG, es capaz de hacer diferenciar las enfermedades mediada por IgG (penfigoide bulloso, PMM, penfigoide gestationis y epidermólisis bullosa adquirida) de las enfermedades mediadas por IgA (dermatitis herpetiforme y la enfermedad linear de IgA) (27).

\section{MANEJO DEL PMM}

Los principales tratamientos disponibles (Esquema 1) son antiinflamatorios, inmunosupresivos o bien una combinación de los dos.

\section{Pacientes con sólo lesiones orales}

Los pacientes de este tipo son los que presentan el decurso mejor desde el punto de vista pronóstico. Se suelen tratar con fármacos tópicos. 


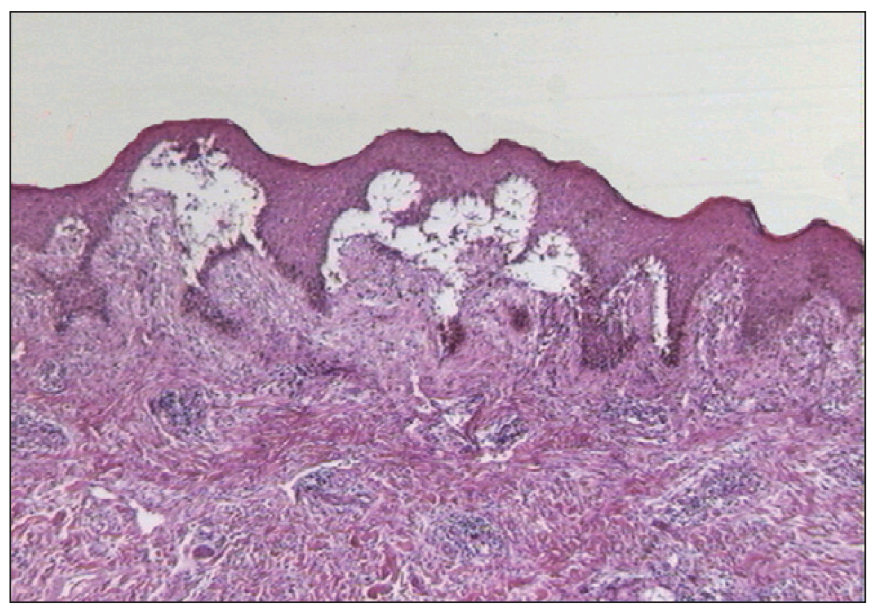

Fig. 5.

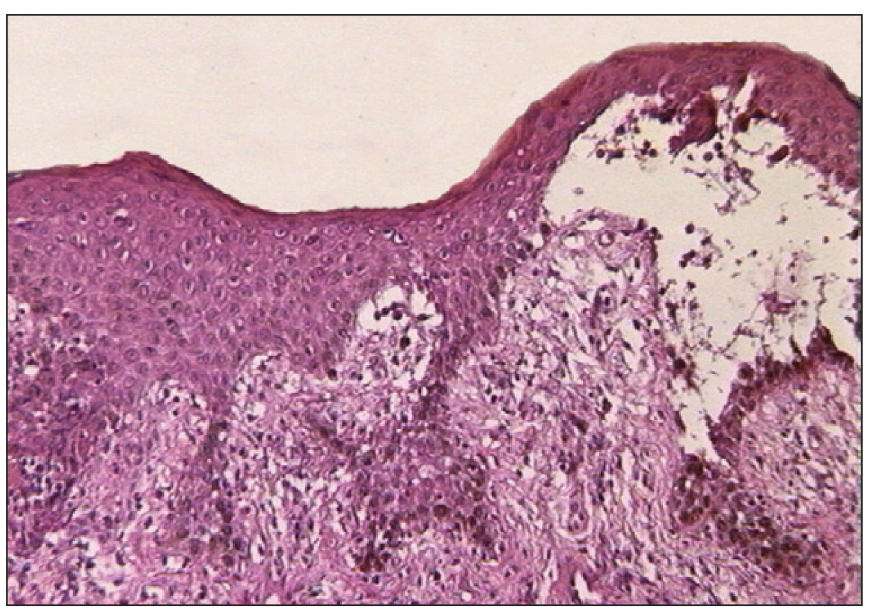

Fig. 6.

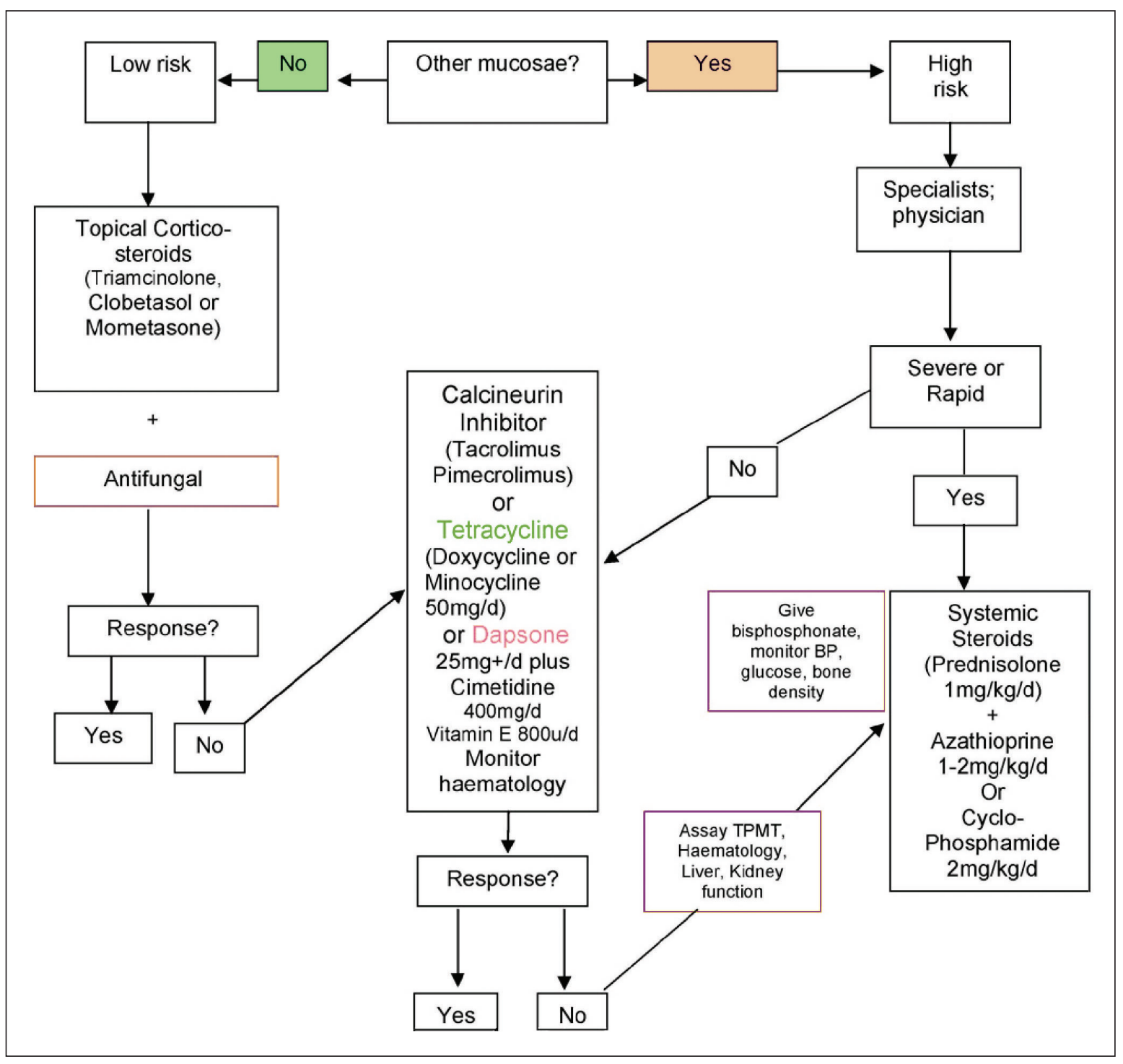

Esquema 1. 


\section{Fármacos de uso tópicos}

Corticosteroides: usualmente se requiere la fluocinocida 0,05\% (2-3 aplicaciones diarias para 9-24 semanas). La triamcinolona $0,1-0,5 \%$ no es adecuada para el tratamiento del PMM, aunque se puede aplicar diluida para tratar lesiones localizadas (29). La candidiasis puede complicar el tratamiento con esteroides, pero se puede prevenir gracias al uso con miconazol o con clorexhidina $(28,29)$.

\section{Antagonistas de la calcineurina}

La Ciclosporina tópica es efectiva para el tratamiento de lesiones orales, pero es muy costosa. El uso tópico de tacrolimo ha sido propuesto, pero su potencial cancerogeneidad ha limitado el uso $(29,30)$.

\section{Pacientes con lesiones no limitadas a la cavidad oral}

Pacientes con lesiones múltiples o bien no localizadas a la cavidad oral, necesitan un tratamiento sistémico más agresivo.

\section{Fármacos sistémicos}

- Dapsone: Es un fármaco capaz de inhibir la adhesión de los neutrófilos y de reducir la síntesis de $\mathrm{PGE}_{2}$.El Dapsone tiene que ser suministrado con discontinuidad, a causa de efectos adversos: anemia hemolítica, supresión de la médula ósea y sobre todo hepatotoxicidad (32). Para minimizar los efectos negativos las pautas a seguir son: $25 \mathrm{mg}$ diario para 3 días, luego $50 \mathrm{mg}$ diarios para 3 días, $75 \mathrm{mg}$ para 3 días luego $100 \mathrm{mg}$ para otros 3 días finalmente enjuagarse con 150 mg diarios hasta el días 17.

- Corticosteroides: el uso de corticosteroides sistémicos con una semivida plasmática breve (prednisone), puede ser muy eficaz. Se aconseja el uso de dosis altas por breve periodos de tiempo. Los corticosteroides sistémicos se pueden asociar a corticosteroides tópicos (clobetasol), para el manejo de la lesión oral.

- Aziatropina o ciclofosfamida: los dos fármacos pueden ser usados, pero ninguno de los dos su- ministrados solamente ha sido demostrado ser suficiente (32). La aziatropina puede inducir colestasis, mientras la ciclofosfamida puede inducir alopecia y cistitis hemorrágicas.

\section{Otros agentes sistémicos}

Un estudio preliminar refiere una respuesta positiva de la lesión al tratamiento condoxyciclina (100 mg diarios para 8 semanas). El uso combinado de tetraciclina y nicotinamida (500 mg hasta $2,5 \mathrm{~g}$ cada día) parece producir una respuesta positiva en casos isolados, aunque se necesitan ensayos clínicos controlados $(33,35)$. En algunos pacientes refractarios a todas las terapias, ha sido usada con éxito la plasmaféresis.

\section{BIBLIOGRAFÍA}

1. Bagan J, Lo Muzio L, Scully C. Mucosal disease series. Number III. Mucous membrane pemphigoid. Oral Dis, 2005;11(4):197-218.

2. Barnadas MA, et al. Clinical, histopathologic, and therapeutic aspects of subepidermal autoimmune bullous diseases with IgG on the floor of salt-split skin. Int J Dermatol, 2001;40(4):268-72.

3. Bohn J, Jonson S, Holst R. Successful treatment of recalcitrant cicatricial pemphigoid with a combination of plasma exchange and cyclophosphamide. Br J Dermatol, 1999:141(3): 536-40.

4. Chan LS. Mucous membrane pemphigoid. Clin Dermatol, 2001;19(6):703-11.

5. Chan LS, et al. Identification and partial characterization of a novel 105-kDalton lower lamina lucida autoantigen associated with a novel immune-mediated subepidermal blistering disease. J Invest Dermatol, 1993;101(3):262-7.

6. Chan LS, et al. Immune-mediated subepithelial blistering diseases of mucous membranes. Pure ocular cicatricial pemphigoid is a unique clinical and immunopathological entity distinct from bullous pemphigoid and other subsets identified 
by antigenic specificity of autoantibodies. Arch Dermatol, 1993;129(4): 448-55.

7. Ciarrocca KN, Greenberg MS. A retrospective study of the management of oral mucous membrane pemphigoid with dapsone. Oral Surg Oral Med Oral Pathol Oral Radiol Endod, 1999;88(2):15963.

8. Damoulis PD, Gagari E. Combined treatment of periodontal disease and benign mucous membrane pemphigoid. Case report with 8 years maintenance. J Periodontol, 2000;71(10): 1620-9.

9. Dayan S, Simmons RK, Ahmed AR. Contemporary issues in the diagnosis of oral pemphigoid: a selective review of the literature. Oral Surg Oral Med Oral Pathol Oral Radiol Endod, 1999;88(4): 424-30.

10. Doan S, et al. Treatment of ocular cicatricial pemphigoid with sulfasalazine. Ophthalmology, 2001;108(9): 1565-8.

11. Egan CA, et al. The immunoglobulin A antibody response in clinical subsets of mucous membrane pemphigoid. Dermatology, 1999;198 (4):330-5.

12. Elder MJ, Leonard J, Dart JK. Sulphapyridinea new agent for the treatment of ocular cicatricial pemphigoid. Br J Ophthalmol, 1996;80(6):54952.

13. Epstein JB, et al. Topical azathioprine in the treatment of immune-mediated chronic oral inflammatory conditions: a series of cases. Oral Surg Oral Med Oral Pathol Oral Radiol Endod, 2001;91(1):56-61.

14. Eschle-Meniconi ME, Ahmad SR, Foster CS. Mucous membrane pemphigoid: an update. Curr Opin Ophthalmol, 2005;16(5):303-7.

15. Eversole LR. Immunopathology of oral mucosal ulcerative, desquamative, and bullous diseases. Selective review of the literature. Oral Surg Oral Med Oral Pathol 1994;77(6):555-71.
16. Fern AI, et al. Dapsone therapy for the acute inflammatory phase of ocular pemphigoid. $\mathrm{Br} \mathrm{J}$ Ophthalmol, 1992;76(6):332-5.

17. Gonzalez-Moles MA, et al. Treatment of severe chronic oral erosive lesions with clobetasol propionate in aqueous solution. Oral Surg Oral Med Oral Pathol Oral Radiol Endod, 2002;93(3): 264-70.

18. Hietanen J, Rantala I, Reúnala T. Benign mucous membrane pemphigoid with linear IgA deposits in oral mucosa. Scand J Dent Res, 1985;93(1): 46-51.

19. Lozada-Nur F, Huang MZ, Zhou GA. Open preliminary clinical trial of clobetasol propionate ointment in adhesive paste for treatment of chronic oral vesiculoerosive diseases. Oral Surg Oral Med Oral Pathol, 1991;71(3):283-7.

20. Lu SY, Chen WJ, Eng HL. Response to levamisole and low-dose prednisolone in 41 patients with chronic oral ulcers: a 3-year open clinical trial and follow-up study. Oral Surg Oral Med Oral Pathol Oral Radiol Endod, 1998;86(4):438-45.

21. Mazzi G, et al. Plasmapheresis therapy in pemphigus vulgaris and bullous pemphigoid. Transfus Apher Sci, 2003;28(1):13-8.

22. McFadden JP, et al. Sulphamethoxypyridazine for dermatitis herpetiformis, linear IgA disease and cicatricial pemphigoid. Br J Dermatol 1989;121 (6):759-62.

23. Mutasim DF, Adams BB. Cicatricial pemphigoid diagnosed by the use of indirect immunofluorescence. J Cutan Med Surg, 2000;4(4):205-7.

24. Rogers RS, 3rd, Seehafer JR, Perry HO. Treatment of cicatricial (benign mucous membrane) pemphigoid with dapsone. J Am Acad Dermatol 1982;6(2): 215-23.

25. Sacher C, et al. Treatment of recalcitrant cicatricial pemphigoid with the tumor necrosis factor alpha antagonist etanercept. J Am Acad Dermatol 2002;46(1):113-5. 
26. Sami N, et al. Intravenous immunoglobulin therapy in patients with ocular-cicatricial pemphigoid: a long-term follow-up. Ophthalmology 2004;111 (7):1380-2.

27. Siegel MA, Balciunas BA. Oral presentation and management of vesiculobullous disorders. Semin Dermatol 1994;13(2):78-86.

28. Sollecito, TP and E. Parisi, Mucous membrane pemphigoid. Dent Clin North Am 2005;49(1):91106, viii.

29. Stoopler ET, DeRossi SS, Sollecito TP. Mucous membrane pemphigoid. Update for the general practitioner. N Y State Dent J 2003;69(8):28-31.

30. Tricamo, $M B$, et al. Periodontal status in patients with gingival mucous membrane pemphigoid. J Periodontol 2006;77(3):398-405.

31. Verdolini R, Cerio R. Autoimmune subepidermal bullous skin diseases: the impact of recent findings for the dermatopathologist. Virchows Arch 2003;443(2):184-93.
32. Vincent, SD,Lilly GE, Baker KA. Clinical, historic, and therapeutic features of cicatricial pemphigoid. A literature review and open therapeutic trial with corticosteroids. Oral Surg Oral Med Oral Pathol 1993;76(4):453-9.

33. Yeh SW, et al. Blistering disorders: diagnosis and treatment. Dermatol Ther 2003;16(3):21423.

34. Roy Villaescusa MJ, Sanz Pérez M, López Fornet P. Penfigoide cicatrizal de largo tiempo de evolución. Av Odontoestomatol 2003;19(4):16775.

35. Nunes R, Lauro Gilberto, Moresco V, Raquel, Marley G, et al. Pénfigo vulgar: caso clínico. Av Odontoestomatol 2005;21(4):189-93.

\section{CORRESPONDENCIA}

N. Discepoli

E-mail:discepoli_nicola@hotmail.it 